J. Math. Biol. (1998) 36: 550-556

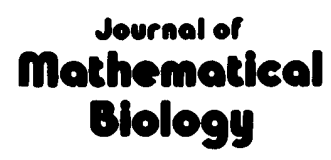

(C) Springer-Verlag 1998

\title{
A simple genetic model with non-equilibrium dynamics
}

\author{
Michael Doebeli ${ }^{1}$, Gerdien de Jong ${ }^{2}$ \\ ${ }^{1}$ Zoology Institute, University of Basel, Rheinsprung 9, CH-4051 Basel, Switzerland \\ e-mail: doebeli@ubaclu.unibas.ch \\ ${ }^{2}$ Population Genetics Group, Department of Plant Ecology and Evolutionary Biology, \\ University of Utrecht, Padualaan 8, $3584 \mathrm{CH}$ Utrecht, The Netherlands \\ e-mail: g.dejong@boev.biol.ruu.nl
}

Received: 5 December 1996/Revised version: 14 November 1997

\begin{abstract}
It is implicit in earlier work that simple population genetic models with constant fertility selection at one locus with two alleles can have nonequilibrium dynamics. But the nature of these dynamics has never been investigated in detail. We show that locally stable 2-cycles occur in these models, which seems to be the simplest genetic models exhibiting such dynamics.
\end{abstract}

Key words: Population genetics - Models - Fertility selection - Gene frequency dynamics

\section{Introduction}

It is well-known that simple ecological models can have complicated dynamics (May 1976). In contrast, simple genetic models usually have stable equilibrium dynamics. More complicated dynamics become likely only if density or frequency dependent fitness functions are used in the population genetic models (May and Anderson 1983; Altenberg 1991; Gavrilets and Hastings 1995; Doebeli and De Jong 1998). The simplest genetic models with constant viability fitness values that exhibit non-equilibrium dynamics are models for two loci with two alleles. Akin (1982) has shown that recombination can cause a Hopf bifurcation and lead to limit cycles in such models when set in continuous time, and Hastings (1981) has reported analogous results for models set in discrete time. With only one locus and two alleles, viability selection models with constant fitness values do not yield non-equilibrium dynamics. This is well-known for the classic model assuming a randomly mating population in Hardy-Weinberg equilibrium (e.g. Roughgarden 1979), but it also holds for models assuming different viability selection in males and 
females and hence not assuming Hardy-Weinberg equilibrium, although the proof for this class of models is rather complicated (Selgrade and Ziehe 1987; Szucs 1991).

A related class of models with one locus and two alleles are fertility selection models, in which constant fitness values are assigned to mating pairs rather than to individual males and females. It is implicit in the early literature on these models that they can exhibit non-equilibrium dynamics (Hadeler and Liberman 1975; Pollak 1978; Feldman et al. 1983). However, the main interest when studying these models had been in the maintenance of genetic polymorphisms (e.g. Clark and Feldman 1986; Christiansen 1989), and interest in gene frequency dynamics was limited to the question of which of the various possible polymorphic equilibria is reached under which conditions. For the record, we explicitly explain in this article how 2-cyclic dynamics arise in fertility selection models with one locus and two alleles. These models are probably the simplest genetic models with non-equilibrium dynamics.

\section{Results}

We assume a genetic system with one locus and two alleles $A$ and $a$. Let $x_{t}$, $y_{t}$ and $z_{t}$ be the frequency of the genotypes $A A, A a$ and $a a$ in the population at the start of generation $t$. Then we consider the model for fertility selection of Hadeler and Liberman (1975), which is summarized in Table 1. The model assumes random mating and Mendelian segregation and is given be the following recurrence equations for the three genotypes (cf. Table 1):

$x_{t+1}=\frac{f_{11} x_{t}^{2}+\frac{1}{2}\left(f_{12}+f_{21}\right) x_{t} y_{t}+\frac{1}{4} f_{22} y_{t}^{2}}{\bar{f}_{t}}$

$y_{t+1}=\frac{\frac{1}{2}\left(f_{12}+f_{21}\right) x_{t} y_{t}+\left(f_{13}+f_{31}\right) x_{t} z_{t}+\frac{1}{2} f_{22} y_{t}^{2}+\frac{1}{2}\left(f_{23}+f_{32}\right) y_{t} z_{t}}{\bar{f}_{t}}$

$z_{t+1}=\frac{\frac{1}{4} f_{22} y_{t}^{2}+\frac{1}{2}\left(f_{23}+f_{32}\right) y_{t} z_{t}+f_{33} z_{t}^{2}}{\bar{f}_{t}}$,

where

$$
\begin{aligned}
\bar{f}_{t}= & f_{11} x_{t}^{2}+f_{22} y_{t}^{2}+f_{33} z_{t}^{2}+\left(f_{12}+f_{21}\right) x_{t} y_{t}+\left(f_{13}+f_{31}\right) x_{t} z_{t} \\
& +\left(f_{23}+f_{32}\right) y_{t} z_{t}
\end{aligned}
$$

is the mean fertility at time $t$. The coefficients $f_{i j}$ are $\geqq 0$ by assumption and are the constant fertilities assigned to matings between phenotypes $i$ and $j$, where $i, j=1,2,3$, and where $1=A A, 2=A a$ and $3=a a$ (see Table 1). To derive our results, we make a number of simplifications in model (1). First of all, only the sums of the fertilities of reciprocal matings enter the equations, hence we can assume that $f_{i j}=f_{j i}, i, j=1,2,3$. Following Hadeler and Liberman (1975), we further assume symmetric fertilities $f_{11}=f_{33}$ and $f_{12}=f_{23}$, which leads to the fertility coefficients shown in Table 2 . Note that 
Table 1. Mating types, fertilities and offspring

\begin{tabular}{|c|c|c|c|c|c|c|}
\hline \multicolumn{2}{|c|}{ Mating type } & \multirow{2}{*}{ Fertility } & \multirow{2}{*}{ Frequency } & \multicolumn{3}{|c|}{ Offspring } \\
\cline { 1 - 3 } male & female & & & $A A$ & $A a$ & $a a$ \\
\hline$A A$ & $A A$ & $f_{11}$ & $x^{2}$ & 1 & 0 & 0 \\
\hline$A A$ & $A a$ & $f_{12}$ & $x y$ & $1 / 2$ & $1 / 2$ & 0 \\
\hline$A A$ & $a a$ & $f_{13}$ & $x z$ & 0 & 1 & 0 \\
\hline$A a$ & $A A$ & $f_{21}$ & $x y$ & $1 / 2$ & $1 / 2$ & 0 \\
\hline$A a$ & $A a$ & $f_{22}$ & $y^{2}$ & $1 / 4$ & $1 / 2$ & $1 / 4$ \\
\hline$A a$ & $a a$ & $f_{23}$ & $y z$ & 0 & $1 / 2$ & $1 / 2$ \\
\hline$a a$ & $A A$ & $f_{31}$ & $x z$ & 0 & 1 & 0 \\
\hline$a a$ & $A a$ & $f_{32}$ & $y z$ & 0 & $1 / 2$ & $1 / 2$ \\
\hline$a a$ & $a a$ & $f_{33}$ & $z^{2}$ & 0 & 0 & 1 \\
\hline
\end{tabular}

Table 2. Symmetric fertility

\begin{tabular}{|c|c|c|c|}
\hline \multicolumn{1}{|c|}{ ○ } & $A A$ & $A a$ & $a a$ \\
\hline \multicolumn{1}{|c|}{$a A$} & $a$ & $b$ & $c$ \\
\hline$A a$ & $b$ & $d$ & $b$ \\
\hline$a a$ & $c$ & $b$ & $a$ \\
\hline
\end{tabular}

one of the equations in model (1) is redundant, since we must always have $x_{t}+y_{t}+z_{t}=1$. Replacing $z_{t}$ by $1-x_{t}-y_{t}$, we finally obtain the following model (Hadeler and Liberman 1975):

$$
\begin{aligned}
x_{t+1} & =\frac{a x_{t}^{2}+b x_{t} y_{t}+\frac{1}{4} d y_{t}^{2}}{\overline{f_{t}}} \\
y_{t+1} & =\frac{b x_{t} y_{t}+b y_{t}\left(1-x_{t}-y_{t}\right)+\frac{1}{2} d y_{t}^{2}+2 c x_{t}\left(1-x_{t}-y_{t}\right)}{\bar{f}_{t}},
\end{aligned}
$$

where

$$
\overline{f_{t}}=a\left(x_{t}^{2}+\left(1-x_{t}-y_{t}\right)^{2}\right)+2 b y_{t}\left(1-y_{t}\right)+2 c x_{t}\left(1-x_{t}-y_{t}\right)+d y_{t}^{2}
$$


is the mean fertility, and where the coefficients $a, b, c$ and $d$ are $\geqq 0$. Hadeler and Liberman (1975) showed that this model can have up to 7 equilibrium points, five of which can be polymorphic with both alleles present at equilibrium. They mentioned that there can be cases in which none of the equilibria are locally stable, but they did not elaborate on the type of non-equilibrium gene frequency dynamics that would occur in such a situation. Referring to Hadeler and Liberman's (1975) observation, Feldman et al. (1983) and Lessard and Castilloux (1995) also mentioned the possibility of non-equilibrium dynamics, but again without going into any details. Pollak (1978) used Hadeler and Liberman's (1975) results to show that mean fitness does not necessarily increase monotonically with time in model (3). He presented a numerical example in which during the course of 5 time steps oscillations of increasing magnitude occurred when the system was initialized close to an unstable equilibrium point. Of course, mean fitness does not increase monotonically if there are oscillations, which was the point that Pollak (1978) wanted to make, and he, too, did not elaborate on the dynamic attractor which the system was approaching under his assumptions. Below we show that the system was in fact moving towards a locally stable 2-cycle.

It is known that if the parameters $c$ and $d$ in system (3) are set to 0 , then the resulting system is equivalent to a one-locus, three-allele viability model (Hadeler and Liberman 1975), which, as a gradient system, cannot produce cycles. In contrast, if $a$ and $b$ are set to 0 in system (3), then only "maximally polymorphic" matings are fertile, i.e. only matings in which either both partners are heterozygotes, or both partners are homozygous for different alleles (cf. Table 2). Moreover, note that the parameter $c$ describes the fertility of matings which only produce offspring whose genotype is different from that of the parents $(A A / a a \rightarrow A a)$, whereas $d$ describes the fertility of matings which produce offspring of all three genotypes $(A a / A a \rightarrow A A, A a, a a)$. Thus $c$ can be viewed as a "destabilizing" parameter, while $d$ can be viewed as a "stabilizing" parameter, and if $a$ and $b$ are 0 , then unstable equilibria can be expected on intuitive grounds if $c$ is "much larger" than $d$. This is the content of the following proposition. If we assume $a=b=0$ in system (3), it immediately follows that the subset $S$ of the state space consisting of those genotype frequencies for which $x=z$, i.e. for which both homozygotes are equally frequent, will be reached from any initial configuration in one time step. Thus the set $S=\left\{(x, y, z) \in \boldsymbol{R}^{3} \mid 0 \leqq x \leqq 1, z=x, y=1-2 x\right\}$ is globally attracting, and we can restrict our analysis to $S$.

Proposition 1. Assume that $a=b=0$ in model (3). Then the set $S$ contains a unique locally stable 2-cycle of system (3) if and only if $c>4 d$.

Proof. Since $z=x$ and $y=1-2 x$ in $S$, model (3) reduces to a 1-dimensional dynamical system on $S$, and it turns out to be most convenient to consider the dynamic variable

$$
w_{t}=\frac{2 x_{t}}{y_{t}},
$$


which can take on values in the interval $[0, \infty]$. Since $a=b=0$, it is easy to see that model (3) leads to the following equation for $w_{t}$ :

$$
\begin{aligned}
w_{t+1} & =g\left(w_{t}\right) \\
& =1-\frac{c w_{t}^{2}}{c w_{t}^{2}+d} .
\end{aligned}
$$

This equation completely describes the dynamics of the system in the set $S$. In particular, locally stable 2-cycles of the variable $w$ correspond to locally stable 2-cycles in the original variables $x, y$ and $z$.

By replacing the parameter $c$ with $c / d$ we get

$$
g(w)=\frac{1}{c w^{2}+1} .
$$

It is easily verified that this function decreases monotonically from 1 to 0 as $w$ varies from 0 to $\infty$. It follows that the equilibrium equation for system (6), $g(w)=w$, has exactly one real, positive solution $w^{*}$. In addition, since the equilibrium equation is a cubic, it also has two complex conjugate solutions.

To find 2-cycles in system (6) we have to find equilibria of the twice iterated map $g^{2}(w)=g(g(w))$. It immediately follows from the chain rule and from the monotonicity of $g(w)$ that $g^{2}(w)$ is a monotonically increasing function on the interval $[0, \infty)$. The equilibrium equation $g^{2}(w)=w$ is a polynomial of order 5 and hence has five solutions. Three of them are the solutions of $g(w)=w$. Therefore, the remaining two are again complex conjugate, and it follows that $g^{2}(w)=w$ has either one or three real, positive solutions.

The monotonicity of $g^{2}(w)$ implies that if there is only one real, positive solution of $g^{2}(w)=w$, then the modulus of the derivative of $g^{2}$ at this solution is $<1$. On the other hand, if there are three real, positive solutions, then the modulus of the derivative of $g^{2}$ is $>1$ at the intermediate solution and $<1$ at the two extremal solutions. It follows that there are exactly two possibilities for the dynamics of system (6):

i) $g^{2}(w)=w$ has only one real, positive solution, which is the equilibrium $w^{*}$ of system (6). This equilibrium is locally stable and globally attractive.

ii) The equilibrium $w^{*}$ is unstable, and $g^{2}(w)=w$ has two other real, positive solutions $w_{1}$ and $w_{2}$ which constitute a stable 2-cycle of system (6) that attracts all initial conditions in $[0, \infty)$ except $w^{*}$.

To see which of these cases holds in dependence of the parameter $c$, we calculate the derivative

$$
\frac{d g}{d w}\left(w^{*}\right)=\frac{-2 c w^{*}}{\left(c w^{* 2}+1\right)^{2}}=-2 c w^{* 3}=-2\left(1-w^{*}\right) .
$$

To obtain the last two equalities in (8) we used the equilibrium condition $w^{*}=g\left(w^{*}\right)$ twice. It follows from the last equality in (8) that $w^{*}$ is unstable if and only if $w^{*}<1 / 2$. Finally, this condition is equivalent to $c>4$ (i.e. to 
$c>4 d$ in the original parametrization) because $c=\left(1-w^{*}\right) / w^{* 3}$. This completes the proof.

Remark. It follows from the proof of Proposition 1 that the equilibrium $w^{*}$ loses its stability through a flip bifurcation, i.e. when the derivative of the function $g$ at $w^{*}$ reaches the value -1 . This type of bifurcation leads to the period-doubling route to chaos in the logistic equation (Drazin 1993). In the present model (6), the period-doubling stops after the first step.

\section{Conclusions}

Fertility selection models with constant fitness values have received quite a lot of attention in the population genetics literature (Bodmer 1965; Hadeler and Liberman 1975; Pollak 1978; Feldman et al. 1983; Abugov 1983; Feldman and Liberman 1985; Liberman and Feldman 1985; Clark and Feldman 1986; Christiansen 1989; Lessard and Castilloux 1995). The main focus was usually on the existence of polymorphic equilibria, and the interest in the dynamics of the models was restricted to the question of which of the equilibria were approached depending on the initial conditions. That fertility selection models with one locus and two alleles can exhibit non-equilibrium dynamics was implicit in the work of Hadeler and Liberman (1975) and of Pollak (1978). However, these authors did not go into details about the nature of the attractors that occur in case that none of the equilibria were locally stable, and neither did later authors who referred to these earlier papers (Feldman et al. 1983; Lessard and Castilloux 1995). Here we showed that under certain conditions system (1) exhibits a locally stable 2-cycle. Of course, the assumptions on the parameters in Proposition 1 are somewhat artificial, but they serve our purpose, which was to fill a gap in population genetic modeling theory and show that simple one locus two alleles models of constant fertility selection can have locally stable 2-cycles. With less restrictive assumptions the analysis would become much more complicated, but we believe that such an analysis, if possible, would not yield any qualitatively new results. An extensive and systematic numerical search of parameter space for the general model (1) indicated that locally stable equilibria and locally stable 2-cycles exhaust all the possible attractors of this system. Thus, Proposition 1 and the Remark following it seem to capture the essence of the dynamic phenomena that can occur in model (1), which seems to be the simplest genetic model exhibiting non-equilibrium dynamics. We conclude by noting that the corresponding one-locus, two-allele fertility model in continuous time does not exhibit non-trivial periodic behaviour (Butler et al. 1982; Hadeler and Glas 1983).

Acknowledgements. We thank two reviewers and K. Hadeler for very helpful comments. Our research was supported by the Netherlands Organisation for Scientific Research (NWO) and the Swiss National Science Foundation. 


\section{References}

Abugov, R.: Genetics of Darwinian fitness. 1. fertility selection. Am. Nat. 121, 880-886 (1983)

Akin, E.: Cycling in simple genetic systems. J. Math. Biol. 13, 305-324 (1982)

Altenberg, L.: Chaos from linear frequency-dependent selection. Am. Nat. 138, 51-68 (1989)

Bodmer, W. F.: Differential fertility in population genetic models. Genetics 51, 411-424 (1965)

Butler, G. J., Freedman, H. I., Waltman, P.: Global dynamics of a selection model for the growth of a population with genotypic fertility differences. J. Math. Biol. 14, 25-35 (1982)

Christinasen, F. B.: The multiple-locus symmetric fertility model. Theor. Pop. Biol. 35, 337-362 (1989)

Clark, A. G., Feldman, M. W.: A numerical simulation of the one-locus, multiple-allele fertility model. Genetics 113, 161-176 (1986)

Doebeli, N., De Jong, G.: Genetic variability in sensitivity to population density affects the dynamics of simple ecological models. Theoretical Population Biology (in press) (1998)

Drazin, P. G.: Nonlinear systems. Cambridge: Cambridge University Press (1993)

Feldman, M. W., Liberman, U.: A symmetric two-locus fertility model. Genetics 109, 229-253 (1985)

Feldman, M. W., Christiansen, F. B., Liberman, U.: On some models of fertility selection. Genetics 105, 1003-1010 (1983)

Gavrilets, S., Hastings, A.: Intermittency and transient chaos from simple frequency-dependent selection. Proc. R. Soc. Lond. B 261, 233-238 (1995)

Hadeler, K. P., Liberman, U.: Selection models with fertility differences. J. Math. Biol. 2, 19-32 (1975)

Hadeler, K. P., Glas, D.: Quasimonotone systems and convergence to equilibrium in a population genetic model. J. Math. Anal. Appl. 95, 297-303 (1983).

Hastings, A.: Stable cycling in discrete-time genetic models. Proc. Natl. Acad. Sci. USA 78, 7224-7225 (1981)

Lessard, S., Castiolloux, A. M.: The fundamental theorem of natural selection in Ewens sense (case of fertility selection). Genetics 141, 733-742 (1995)

Liberman, U., Feldman, M. W.: A symmetric two-locus model with viability and fertility selection. J. Math. Biol. 22, 31-60 (1985)

May, R. M.: Simple mathematical models with very complicated dynamics. Nature 261, 459-467 (1976)

May, R. M., Anderson, R. M.: Epidemiology and genetics in the coevolution of parasites and hosts. Proc. R. Soc. Lond. B 219, 281-313 (1983)

Pollak, E.: With selection for fecundity the mean fitness does not necessarily increase. Genetics 90, 383-389 (1978)

Roughgarden, J.: Theory of population genetics and evolutionary ecology: an introduction. New York: MacMillan (1979)

Selgrade, J. F., Ziehe, M.: Convergence to equilibrium in a genetic model with differential viability between the sexes. J. Math. Biol. 25, 477-490 (1987)

Szucs, J. M.: Selection at a diallelic autosomal locus in a dioecious population. J. Math. Biol. 29, 693-713 (1991)

Note added in proof. Results similar to our Proposition 1 have been obtained independently by K. Josic (Local bifurcations in the symmetric model of selection with fertility differences. 1997. Journal of Theoretical Biology 189, 291-295). 